\title{
OMOY: A Handheld Robotic Gadget that Shifts its Weight to Express Emotions and Intentions
}

\author{
Yohei Noguchi \\ University of Tsukuba \\ Tsukuba, Japan \\ noguchi@ftl.iit.tsukuba.ac.jp
}

\author{
Fumihide Tanaka \\ University of Tsukuba \\ Tsukuba, Japan \\ fumihide.tanaka@gmail.com
}

\begin{abstract}
A robotic gadget that is equipped with a movable weight inside its body is developed. By controlling the movement of the internal weight together with other robotic behaviors such as hand gestures or speech dialogues, it is expected that emotional and/or intentional messaging between users is enhanced. To gain knowledge for designing effective weight shifts, an elicitation study was conducted to investigate how users holding this gadget in their hand interpreted its 36 weight shift patterns generated by setting four basic movement parameters (target position, trajectory, speed, and repetition). Results present mappings between these parameters and the emotional perception of the users. Furthermore, specific weight shift patterns that can express certain human emotions and intentions are revealed. These findings will be useful for designing effective weight shifts to enhance emotional and intentional messaging between users. This study attempts to open a new dimension for the expression capability of robotic gadgets.
\end{abstract}

\section{Author Keywords}

Social mediator robot; communication agent; weight shift; expression; emotional/intentional messaging; elicitation study.

\section{CCS Concepts}

-Human-centered computing $\rightarrow$ Haptic devices; User studies; Human computer interaction (HCI);

\section{INTRODUCTION}

Robotic gadgets are becoming increasingly available for many people. In addition to smart speakers, more robotic gadgets, such as robot phones, have already been introduced to the market. For example, RoBoHoN [7], produced and distributed by a major electronics company, has a small humanoid appearance and mediates human phone calls via robot behaviors, such as gestures and speech dialogues.

However, the communication capability of robots remains insufficient in many aspects. Numerous studies have shown that people lose interest in their robots after the novelty effect

Permission to make digital or hard copies of all or part of this work for personal or classroom use is granted without fee provided that copies are not made or distributed for profit or commercial advantage and that copies bear this notice and the full citation on the first page. Copyrights for components of this work owned by others than ACM must be honored. Abstracting with credit is permitted. To copy otherwise, or republish, to post on servers or to redistribute to lists, requires prior specific permission and/or a fee. Request permissions from permissions@acm.org.

CHI '20, April 25-30, 2020, Honolulu, HI, USA.

(C) 2020 Copyright is held by the owner/author(s). Publication rights licensed to ACM. ACM ISBN 978-1-4503-6708-0/20/04 ...\$15.00.

DOI: http://dx.doi.org/10.1145/3313831.3376775
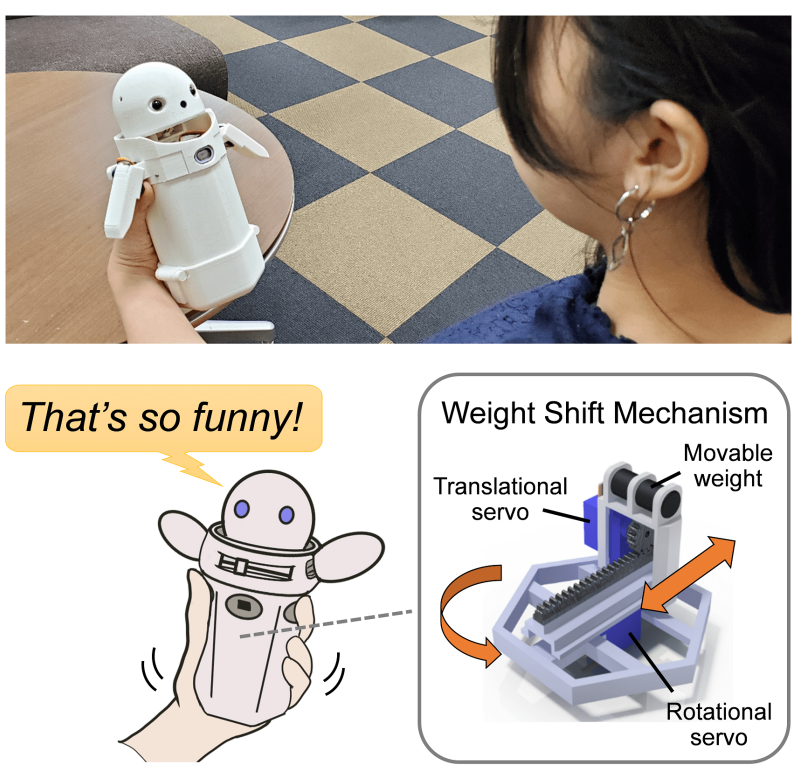

Figure 1. The robotic gadget, named OMOY, developed in this study can exhibit various weight shifts to express emotions and intentions. A movable weight is implemented inside its body and is controlled by the translational and rotational mechanisms determined by four parameters (target position, trajectory, speed, and repetition). By exhibiting appropriate weight shifts together with gestures or speech dialogues, emotional and intentional messaging between users is expected to be enhanced.

disappears $[13,44,9]$. The RoBoHoN was also reported to be unable to sustain users' interest [29]. Overall, the manner in which the robot's messages are delivered tends to be flat, and people quickly lose interest. This issue is particularly serious for situations in which important messages must be delivered.

To address this issue, in this paper, we introduce the concept of weight shifts in robot communications (Figure 1). We developed a handheld robotic gadget equipped with a movable tungsten weight inside its body. By controlling the translational and rotational motion of the weight via four parameters (target position, trajectory, speed, and repetition), the gadget can exhibit weight shifts to the user who holds the robot.

By combining weight shifts with other robotic behaviors, such as hand gestures, facial expressions, and speech dialogues, the emotional elements of messages are expected to be enhanced, thus becoming more engaging to users. However, due to a lack of knowledge regarding the manner in which humans perceive weight shifts, appropriately designing the weight 
shifts was difficult. Therefore, we conducted an elicitation study to investigate how users interpret various weight shifts. Specifically, we prepared a total of 36 weight shift patterns based on four basic parameters, as mentioned above, and we then questioned users regarding their perceptions of each weight shift pattern. In this study, we focused on testing the weight shifts, i.e., other robot behaviors such as gestures or speech were not used. In the interviews, we measured the users' perceived haptic stimuli, perceived emotional states, and perceived intentions of the robotic gadget. We then applied an elicitation procedure in which the users were asked to verbalize their perception by using a questioning methodology proposed by [32]. We analyzed the obtained results to have design recommendations for the weight shifts. Factor and clustering analyses provided us with useful knowledge with respect to mappings between the users' emotional/intentional perceptions and the four basic parameters.

In summary, the contributions of this study are as follows:

- The hardware design of a robotic gadget, named OMOY, that is capable of shifting its weight in a controlled manner.

- An elicitation study investigating how users perceive and verbalize various weight shift patterns.

- Design recommendations for weight shifts that presumably be effective for enhancing messaging between users.

\section{RELATED WORKS}

Vibrotactile (touch and vibration) stimuli have been used for communication purposes since Geldard first discussed this topic in the 1950s [12]. A three-fingered tactual display called Tactuator was an early device that was able to transmit complex information through vibrotactile stimuli [43]. Recently, ComTouch expanded this concept to remote interpersonal communication using mobile phones [6]. Réhman studied in detail the use of vibrotactile rendering in mobile phones [34]. Vibration motors were used to render vibrotactile patterns that expressed human emotions [35, 36]. Similar methods have also been applied for the emotional expressions of game avatars [1]. All of these works used vibrotactile stimuli generated by vibration motors. By contrast, the present study explores stimuli caused by weight shifts implemented by the combination of two (translational/rotational) motions of a weight. In this paper, we will report the study results that are characteristic of such weight shifts.

Handheld devices equipped with weight shift functions have been explored in VR (virtual reality) or game applications. TorqueBAR [42] is an ungrounded force feedback device having a weight moving horizontally (1D) between left and right. Possible applications for the device such as video games and navigation were discussed based on a user study [42]. WeightShifting Mobile [15] is a mass-shift based system for haptic actuation in mobile phones. It was tested whether users were able to determine the position of a weight inside the device [15]. To change both the weight and volume of a device, liquid metal transfer by using a bi-directional pump was proposed [28]. This method was able to be applied for making a miniature solar system and motion control [28]. Shifty [50] is a rod-shaped

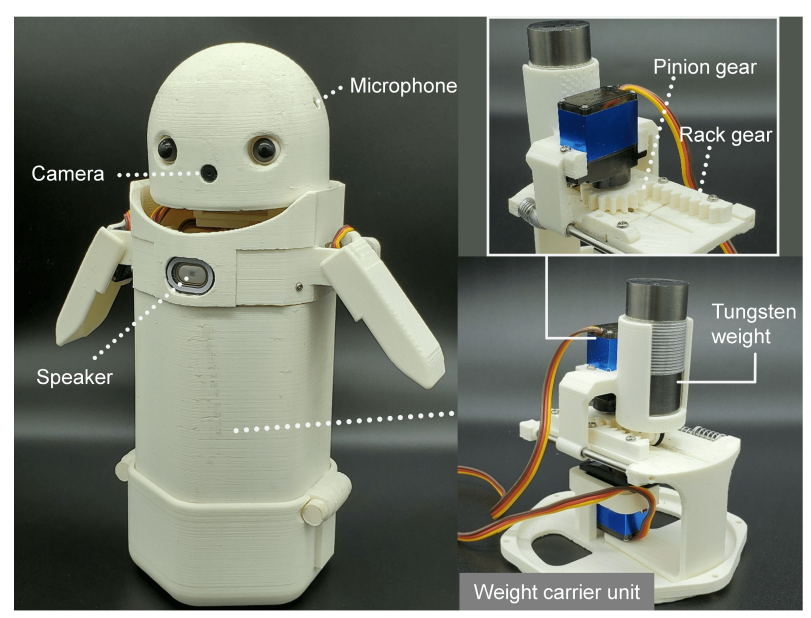

Figure 2. Prototype of OMOY. A $250 \mathrm{~g}$ tungsten weight is attached to the weight carrier unit, which allows the weight to move along a 2D planar space. For translational motion, the mechanism was actuated by a servo motor through rack and pinion gears.

VR controller that can change its internal weight distribution to enhance object perception. It also has a weight moving in 1D to enhance the user perception of virtual objects changing in shape (length). Transcalibur [38] is a transformable VR controller that can render a $2 \mathrm{D}$ shape by changing its mass properties on a 2D planar area. A computational model to control the movement of the controller was obtained from a user study [39]. Compare to these previous works, the novelty part of the present study exists in targeting emotional and intentional messaging between a device and its user by introducing more complex weight shift patterns in 2D space than these previous works.

Emotional expression is a major research topic in robotics. Facial expressions have been most commonly explored since the 1990s [14]. SEER, which was recently demonstrated at SIGGRAPH 2018 Emerging Technologies, has state-of-the-art capability in its facial expressions [46]. The whole body movement [25], sounds [17], colors [45], vibration motors [40], and their combinations [21, 40] have also been used for expressing robotic emotions. In addition, haptic factors such as skin temperature [27, 33, 2], skin texture [16], and SwarmHaptics [20] are discussed within the context of robotic emotional expression. However, the use of weight shifts was unexplored in social robotics.

\section{HARDWARE DESIGN}

The overall hardware specifications of the robotic gadget, named OMOY, are illustrated in Figure 2. The dimensions of OMOY are approximately $240 \mathrm{~mm}(\mathrm{H}) \times 125 / 230 \mathrm{~mm}(\mathrm{arm}$ closed/arm open) (W) $\times 95 \mathrm{~mm}(\mathrm{D})$, and its total weight is $720 \mathrm{~g}$. To minimize the influence of prior knowledge or experience with other commercial robots, OMOY was designed with a simple appearance. Following the information reported in [22], its facial appearance (eyes) was designed to render the robot suitable for home use. For prototyping, the eyes were represented as plastic glossy black buttons that are often used for stuffed animals. The mechanical designs of the weight carrier unit that controls the weight shifts and the robotic unit 
that controls the voice and other robotic behaviors will be explained in the following sections.

\section{Weight Carrier Unit}

The body exterior of OMOY forms a regular hexagon. The inside shapes cylindrical with a diameter of $80 \mathrm{~mm}$. In that hollow space, $250 \mathrm{~g}$ of tungsten weight $(\phi 20 \times l 42 \mathrm{~mm})$ is implemented. Tungsten has a large specific weight $\left(19.3 \mathrm{~g} / \mathrm{cm}^{3}\right)$, high chemical stability, and a reasonable price.

When a user grips the gadget, two types of friction are applied to the skin surface: a frictional force against gravity and a frictional force that stops the rotation. Assuming that the user holds the robot at two points, the thumb and middle finger, and assuming that the static friction coefficient $\mu$ for the robot surface and human skin is uniform, the force $F$ that the user should apply perpendicular to the robot to fix its position and posture is derived as below.

$$
F=\frac{g}{2 \mu}\left\{m\left(1+\frac{3}{D} l\right)+M\right\} .
$$

( $m$ : mass of the weight, $M$ : mass of the gadget excluding the mass of the weight, $l$ : inner radius of the gadget, $D$ : diameter of the human-gadget contact area) In addition, according to Weber's law in psychophysics, the human differential threshold on the skin for pressure stimulus is 1 of 7 [26]. Based on these relationship, we simulated $F$ for different parameters $m$, $M, l$, and $D$, and concluded that the weight should be $250 \mathrm{~g}$ or more for implementing the gadget. The tungsten weight is attached to a translational and rotational mechanism that allows the weight to move along a 2D planar space.

For the translational mechanism, rack and pinion gears were used, which were 3D-printed with ABS resin. The pinion gear is connected to a Tower Pro MG92B servo motor $(3.1 \mathrm{kgf} \cdot \mathrm{cm}$ output for a $5.0 \mathrm{~V}$ input) and conveys its torque to the rack gear. The total length of the rack is $47.6 \mathrm{~mm}$, and the weight can be moved from the origin (center of the robot) to the inner wall of the robot for each gear pitch of $3.86 \mathrm{~mm}$. The translational weight shifts are guided by two metal shafts $(\phi 6 \mathrm{~mm})$ next to the servo, rack, and pinion gears (path of the weight), and coil springs are applied to these metal shafts for shock absorption. In addition, a wheel is attached under the weight to ensure that the movements are smooth. The total weight of the part assembly (including the tungsten weight, servo motor, pinion gear, wheel, and weight-servo joint) moved by the translational mechanism is $280 \mathrm{~g}$.

For the rotational mechanism, the MG92B servo motor was used. This motor is not only vertically connected to the translational mechanism but is also fixed to the robot exterior through the base of the weight carrier unit. In this prototype, the weight is capable of rotating in any direction between 0 and 180 degrees on the front side of the robot.

\section{Robotic Unit}

OMOY has two DOFs on its neck (head nod and swing) and one DOF on each shoulder (right/left), and a Tower Pro SG92R servo motor is introduced to each joint $(2.5 \mathrm{kgf} \cdot \mathrm{cm}$ output for $5.0 \mathrm{~V}$ input). An Arduino Nano microcontroller board is used

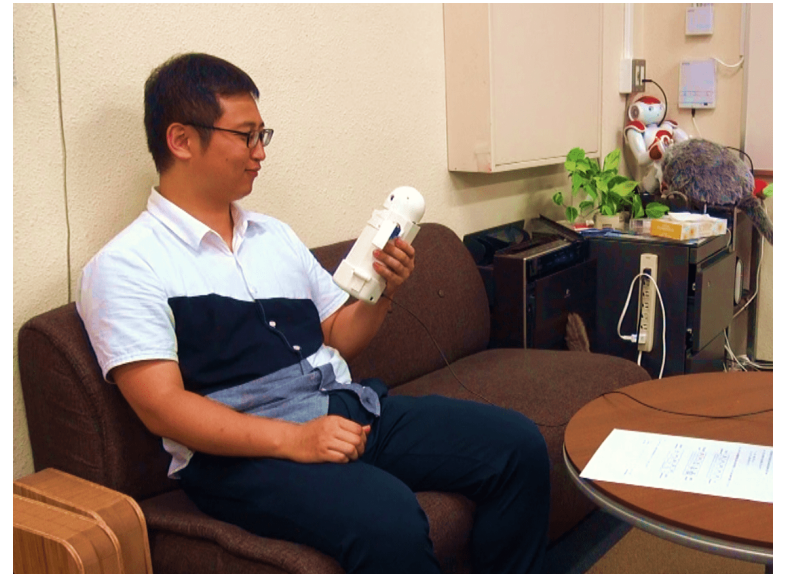

Figure 3. Elicitation study: Each participant was exhibited 36 patterns of weight shifts by OMOY in his/her hand. An experimenter sat in front of the participant for the interviews.

to control these servo motors and is serially connected via a USB to a small built-in CPU board (Raspberry Pi Zero). The CPU board is also connected to a microphone array (Seeed Studio ReSpeaker 2-Mics Pi HAT) and a camera module (Raspberry Pi Camera Module V2), both of which are embedded in the robot's head. The "ear holes" are located on both sides of the robot head, and the camera hole is located on the face. In addition, a micro speaker module is mounted on the chest.

We envision that OMOY will be used as a social mediator that connect humans by delivering messages. Thus, we implemented the capability for voice interactions by using a Google Assistant API. Messages can be exchanged through an HTTPS web server that was developed to manage messages posted and requested from the robots. A Python script runs on the built-in CPU board, which provides speech, web server access, and high-level robot control, and the Arduino Nano controlled the servo motors to provide weight shifts and other robotic behaviors. The power for the robot was supplied by $5 \mathrm{~V} \mathrm{DC}$ via a micro USB and can also be supplied by mobile batteries often used for smartphones or smart devices. Thus, the robot can be operated in a portable manner.

\section{ELICITATION STUDY}

To design the effective use of weight shifts, we need to know about the perception of users towards weight shift patterns. To obtain such knowledge, we conducted an elicitation study. A total of 36 weight shift patterns generated by setting four movement parameters (target position, trajectory, speed, and repetition) explained in below were exhibited to 18 participants. Each participant tested these 36 weight shift patterns one by one, and then was interviewed by an experimenter (Figure 3). Because we wanted to know first about the effects of weight shifts, in this study, we focused on the weight shifts, i.e., other behavioral functions such as head/arm movements or speech dialogues were not used.

Considering the future use case of OMOY as a social mediator that was mentioned in the previous section, it was required for OMOY to be able to convey the "importance" of a message. Following psychology literature [18] suggesting that the 
abstract concept of importance is grounded in bodily experiences of weight, we aimed to create weight shifts in which users could feel as if OMOY gained its weight when reporting important messages. During pilot trials repeated within researchers, we found that movements toward the front side were easier to perceive a weight gain than them toward the back side. It was presumably because the front side was more apart from users' hand (thus made them feel more weight by lever principle) than the back side. Therefore, we decided to explore weight shift patterns mainly in the front side area.

In determining movement parameters, we also referred to [20] in which a haptic interaction case involving small mobile robots having 2D-spacial and 1D-rotational degrees of freedom were discussed. More specifically, movement parameters such as trajectory, speed, and repetition were chosen by referring to the approach taken in [20]. Then, we repeated pilot trials again to finalize the attributes of these parameters so that we had a set of representative weight shifts.

\section{Four Movement Parameters}

\section{Target position}

The target position is the destination of a weight movement. We explored three target positions: front, ua_side, and op_side, as shown in Figure 4. The front indicates the front side of OMOY facing toward the user holding it from the back with his/her non-dominant hand (see Figure 3). ${ }^{1}$ The ua_side (user arm side) indicates the side that is near to the forearm of the user who holds OMOY, whereas the op_side indicates the opposite side of the ua_side.

\section{Trajectory}

The shapes of the weight trajectories were manipulated in three ways shown in Figure 5: one direct path (traveling straight from the origin to a target position) and two indirect paths (traveling to a target position while passing through other positions). When the indirect paths are taken, a force is produced by the translations and a centrifugal force is produced by the rotations. After the target position is reached, the weight returns to the origin.

\section{Speed}

Two speeds were explored: fast and slow. Fast denotes the maximum speed at which the servo motor can move the weight, and slow denotes $30 \%$ of the maximum speed. When the weight moves in the fast mode, the peak acceleration was observed as $6 \mathrm{~m} / \mathrm{s}^{2}$ for translation and $9 \mathrm{~m} / \mathrm{s}^{2}$ for rotation, as measured by a Kionix KXR94-2050 accelerometer. In the slow movements, acceleration peaks were observed at $2 \mathrm{~m} / \mathrm{s}^{2}$ for translation and $3 \mathrm{~m} / \mathrm{s}^{2}$ for rotation. The speed parameter was controlled by VarServoSpeed.h imported from Arduino library.

\section{Repetition}

Two types of repetition were explored: single motion and repetitive motion. For the single motion, the weight is moved only once to the target position while tracing a trajectory.

\footnotetext{
${ }^{1}$ All data were converted such that the relative positional relationship between OMOY and each participant's hand was constant across all participants (right-handed and left-handed).
}

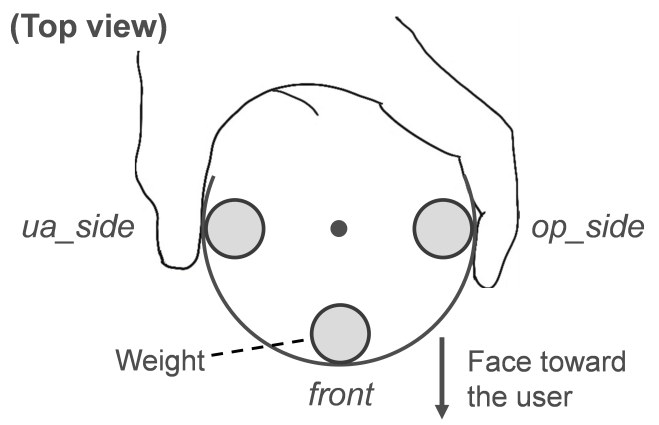

Figure 4. Three target positions of the weight.

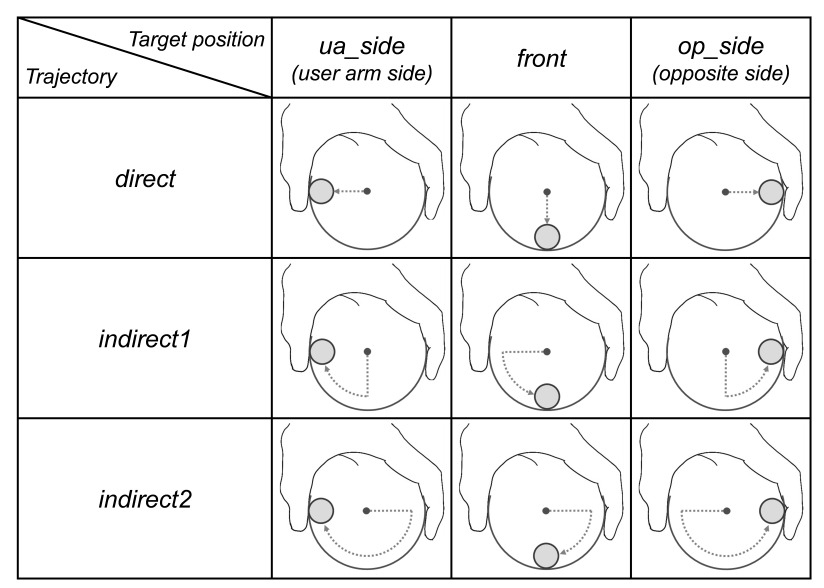

Figure 5. Three target positions $\times$ three trajectories of the weight.

In the repetitive motion, the weight performs one-and-a-half round trips along the trajectory as follows: 1) travel to the target position, 2) return to the origin through the same path, and 3) return again to the target position. ${ }^{2}$

\section{Participants}

Eighteen participants (14 males, 4 females, ages: $21-36$ years) were recruited in the University of Tsukuba. None of the participants had mental disorder, arm/hand injury, or any other issues that could affect their performance during the experiments. Each participant was paid 1,660 JPY ( $120 \mathrm{~min})$ for their cooperation. The study protocol was approved by the Research Ethics Committee in the Faculty of Engineering, Information, and Systems in the University of Tsukuba (2019R317), and all participants provided informed consent.

\section{Data Collection (Measurements)}

For each weight shift pattern, we collected users' perceptions through interviews. Questions were presented through a questionnaire sheet and was orally answered by the participants.

\footnotetext{
${ }^{2}$ In designing weight shifts patterns, two time delays were incorporated as follows. A $5.0 \mathrm{~s}$ pause was made at the end of each movement, i.e., between the time at which the weight lastly reached the target position and the time at which it began to return to the origin. A $0.3 \mathrm{~s}$ of transaction time was used for translation-rotation movements in the indirect path and for translation-translation and rotation-rotation movements in the repetitive motion.
} 


\section{Perceived haptic stimuli}

We asked if the participants felt the weight moving inside the robot (prior to the trials, the participants had been informed that the robot would perform weight shifts inside its body, as will be described in the procedure section). Each participant answered either " 1 : Yes, I felt." or " 2 : No, I didn't feel." For this study, this question item was used to ensure that the participants could perceive each haptic stimulus.

\section{Perceived emotional states}

The perceived emotional state of OMOY during the weight shifts was evaluated. For this measurement, we used a ninepoint Self-Assessment Manikin [3], which is a visual scale of three dimensions in the pleasure, arousal, and dominance (PAD) model [24]. The participants were asked to "Please guess the emotional states of the robot." and were presented with the manikin illustrations.

\section{Perceived intentions}

It is expected that participants perceive not only the emotional states but also the intentions of OMOY exhibiting weight shifts. Thus, we collected user descriptions of the OMOY's intentions during weight shifts by using an elicitation interview technique. To this end, we first asked the participants whether they perceived any intentions from the weight shifts of the robot. In the field of interaction between the human and computer agents, the intentions of agents are discussed in three dimensions: purposefulness, interaction, and animateness [10]. Following this concept, we revised a questionnaire item for measuring the purposefulness dimension and asked the participant "How strongly was OMOY trying to express something to you?" Each participant answered the question over a range of " 1 : Not at all." to "9: Very strongly." Then, the elicitation process was carried out in accordance with the score from the prior question. For the participants who perceived intentions (who marked a value 6 or greater on the previous question), we asked "What words would you use to describe what the robot is trying to express to you?" For the other participants (who marked a value of less than 6 on the previous question), we asked "What words would you use to describe how it felt on your hand, if at all?" Furthermore, the following questions were asked to all the participants: "Was there anything else you felt or thought or are there any sensations or pictures that come in your minds?" and "Is there any additional comment related with this trial?" These question items were based on the explicitation interview technique reported in [32].

\section{Procedure}

The trials were conducted under face-to-face conditions between an experimenter and each of the participants. Initially, each participant was given an overall introduction of this study, and their consent was requested, followed by instructions on how to interact with OMOY. It was introduced to the participants as " $a$ hand-held communication robot that is equipped with a movable weight inside the body," and we informed them that they would receive various haptic stimuli caused by the weight shifts. The participants were also instructed that in this trial OMOY did not speak nor move its hands, thus they paid attention to the weight shifts. In addition, they were instructed to hold OMOY from the back with his/her non-dominant hand (similar to the position for holding a smartphone) and not to tilt OMOY by more than 45 degrees (to prevent it from lying down). We disclosed all question items used in the interview process in advance, and then, 36 weight shift patterns were presented in a random order. The experimenter produced each stimulus by sending commands to OMOY through a laptop. Each pattern had a duration of less than $10 \mathrm{~s}$ and was presented twice. If the participant was ambivalent regarding his/her answer, we presented as many additional stimuli as the participant desired. At the end of each stimulus, the experimenter interviewed the participant regarding their perceptions of OMOY, with instructions to leave it on a table. ${ }^{3}$ We held a break with a duration of a few minutes every $30 \mathrm{~min}$, and all procedures were completed within 120 minutes. All of the experimental procedures, including each trial and interview, were videotaped.

\section{RESULTS AND DISCUSSION}

\section{Perceived Haptic Stimuli (Manipulation Check)}

We checked whether each stimulus was well-perceived by the participants. For 28 of the 36 patterns, all participants reported that the weight was moving. For 5 patterns, 1 participant did not recognize the stimulus, and 2 participants for 1 pattern and 3 participants for 2 patterns reported that they did not feel the weight shifts. The conditions in which 2 or 3 participants did not perceive the stimuli were front-indirect2-fast-single, op_side-direct-slow-single, and ua_side-direct-slow-single, denoting [target position]-[trajectory]-[speed]-[repetition]. In the subsequent analyses, the data of participants who did not perceive the stimulus were excluded (in corresponding conditions).

\section{Parameter Results}

We tested the effects of each parameter on the perceptions of emotional states (pleasure, arousal, and dominance) and intentions (purposefulness). Because the shapes of the trajectory depend on the target positions, $3 \times 2 \times 2$ ANOVA was performed for each target position, with the factors of trajectory, repetition, and speed. 4 All significant results are summarized in Table 1. Other detailed statistical results such as the mean values and standard deviations for each condition are provided in Auxiliary Material for this paper.

\section{Pleasure}

For the target position front, a significant main effect of speed was observed; participants perceived more pleasure from the robot with fast weight shifts than for slow weight shifts.

For the three-way interaction confirmed with this target position, we performed a simple interaction test as a post-hoc comparison. The results indicated that the perceived pleasure for front-direct-fast-repetitive was higher than that for frontdirect-slow-repetitive $(p=.007)$. Moreover, the perceived

\footnotetext{
${ }^{3}$ Participants were also informed that the weight shifting would stop for $5.0 \mathrm{~s}$ when each stimulus was completed. The participants were asked to answer the questions based on their feelings during continuous weight moving, excluding this long pause.

${ }^{4}$ Repeated-measure metrics were not suitable for this analysis because missing data were removed through the manipulation check, with no answers from the participants.
} 
Table 1. Parameter results on perceived emotional states and intentions. The combinations of parameters having statistical significance are listed. 'Traj,' 'spd,' and 'rept' denote trajectory, speed, and repetition.

\begin{tabular}{|c|c|c|c|c|}
\hline \multicolumn{5}{|c|}{ Pleasure } \\
\hline \multirow[t]{2}{*}{ front } & spd & $F(1,193)=12.2$ & $p=.001$ & $\eta_{p}^{2}=.059$ \\
\hline & traj $\times$ spd $\times$ rept & $F(2,193)=3.58$ & $p=.030$ & $\eta_{p}^{2}=.036$ \\
\hline \multicolumn{5}{|l|}{ Arousal } \\
\hline \multirow[t]{3}{*}{ front } & traj & $F(2,193)=28.9$ & $p<.001$ & $\eta_{p}^{2}=.23$ \\
\hline & rept & $F(1,193)=11.0$ & $p=.001$ & $\eta_{p}^{2}=.054$ \\
\hline & traj $\times$ rept & $F(2,193)=5.32$ & $p=.006$ & $\eta_{p}^{2}=.052$ \\
\hline \multirow[t]{3}{*}{ ua_side } & traj & $F(2,184)=18.9$ & $p<.001$ & $\eta_{p}^{2}=.17$ \\
\hline & spd & $F(1,184)=34.8$ & $p<.001$ & $\eta_{p}^{2}=.16$ \\
\hline & rept & $F(1,184)=29.1$ & $p<.001$ & $\eta_{p}^{2}=.14$ \\
\hline \multirow[t]{5}{*}{ op_side } & traj & $F(2,191)=6.77$ & $p=.001$ & $\eta_{p}^{2}=.066$ \\
\hline & spd & $F(1,191)=24.3$ & $p<.001$ & $\eta_{p}^{2}=.11$ \\
\hline & rept & $F(1,191)=24.5$ & $p<.001$ & $\eta_{p}^{2}=.11$ \\
\hline & traj $\times$ rept & $F(2,191)=3.34$ & $p=.038$ & $\eta_{p}^{2}=.034$ \\
\hline & spd $\times$ rept & $F(1,191)=5.58$ & $p=.019$ & $\eta_{p}^{2}=.028$ \\
\hline \multicolumn{5}{|c|}{ Dominance } \\
\hline \multirow[t]{3}{*}{ front } & traj & $F(2,193)=21.1$ & $p<.001$ & $\eta_{p}^{2}=.18$ \\
\hline & rept & $F(1,193)=5.92$ & $p=.016$ & $\eta_{p}^{2}=.030$ \\
\hline & $\operatorname{traj} \times$ spd & $F(2,193)=4.51$ & $p=.012$ & $\eta_{p}^{2}=.045$ \\
\hline \multirow[t]{2}{*}{ ua_side } & traj & $F(2,184)=3.46$ & $p=.033$ & $\eta_{p}^{2}=.036$ \\
\hline & spd & $F(1,184)=5.15$ & $p=.024$ & $\eta_{p}^{2}=.027$ \\
\hline \multirow[t]{3}{*}{ op_side } & traj & $F(2,191)=4.13$ & $p=.018$ & $\eta_{p}^{2}=.04 \overline{1}$ \\
\hline & spd & $F(1,191)=15.8$ & $p<.001$ & $\eta_{p}^{2}=.076$ \\
\hline & rept & $F(1,191)=4.89$ & $p=.028$ & $\eta_{p}^{2}=.025$ \\
\hline \multicolumn{5}{|c|}{ Intentions } \\
\hline front & rept & $F(1,193)=6.37$ & $p=.012$ & $\eta_{p}^{2}=.032$ \\
\hline \multirow[t]{2}{*}{ ua_side } & traj & $F(2,184)=4.16$ & $p=.017$ & $\eta_{p}^{2}=.0 \overline{4}$ \\
\hline & rept & $F(1,184)=6.06$ & $p=.015$ & $\eta_{p}^{2}=.032$ \\
\hline \multirow[t]{2}{*}{ op_side } & traj & $F(2,191)=4.60$ & $p=.011$ & $\eta_{p}^{2}=.046$ \\
\hline & rept & $F(1,191)=19.5$ & $p<.001$ & $\eta_{p}^{2}=.093$ \\
\hline
\end{tabular}

pleasure for front-indirect1-fast-repetitive was higher than that for front-indirect1-slow-repetitive ( $p=.007)$. No significant effects were found for the other two target positions.

\section{Arousal}

A significant main effect of the trajectory for each target position was observed. Post-hoc comparisons using the Tukey HSD indicated that for the direct path to each target position, the perceived arousal was significantly lower than the two indirect paths (all $p<.05$ ).

A significant main effect of repetition was also observed for each target position: when the weight moved in a repetitive motion, OMOY was perceived as having a higher arousal state than when the weight moved in a single motion.

In addition, the effects of speed were significant with two target positions $u a \_s i d e$ and $o p \_s i d e$ : the faster the weight moved, the higher the arousal state perceived as the weight moved to these target positions.

For the target positions front and op_side, an interaction between the trajectory and repetition was observed. A post-hoc comparison indicated that for the indirect paths, arousal was strongly perceived when the weight repetitively moved compared with a single movement: front-indirectl $t(193)=2.84$, $p=.005, d=.66 ;$ front-indirect $2 t(193)=3.56, p<.001$, $d=1.07 ;$ op_side-indirect1 $t(191)=2.29, p=.023, d=.48$; and $o p \_s i d e$-indirect $2 t(191)=5.01, p<.001, d=1.14$.

For the target position op_side, an interaction between the speed and repetition was observed. A post-hoc comparison indicated that the fast repetitive weight shifts were associated with higher arousal states than the slow repetitive $(t(191)=$ $4.24, p<.001, d=.92)$ and fast single $(t(191)=4.28, p<$ $.001, d=1.02$ ) weight shifts.

\section{Dominance}

A significant main effect of the trajectory for each target position was observed. Post-hoc comparisons using the Tukey HSD indicated that for the direct path to the front position, a lower dominance was perceived compared with the two indirect paths (all $p<.001$ ). In addition, when the weight moved to the $u a \_$side position while passing the $o p_{-}$side position (ua_side-indirect2), a higher dominance was perceived compared with the direct path $(p=.035)$. When the weight moved to the $o p \_s i d e$ position by passing the ua_side position (op_side-indirect2), the participants perceived a higher dominance compared with the other two paths (direct $p=.050$, $o p \_$side-indirectl $p=.025$ ).

The effect of speed was also significant for ua_side and $o p \_s i d e$ positions; the faster the weight moved, the higher the perceived dominance was.

In addition, the repetition was significant for two target positions front and $o p \_s i d e$; a higher dominance was perceived when the weight repetitively moved compared with single motions.

For the front target position, an interaction was observed between the trajectory and speed. A lower dominance was perceived when the weight slowly moved along the direct path compared with fast movements $(t(193)=2.98, p=.003$, $d=.70)$.

\section{Intentions}

A significant main effect of the repetition for each target position was observed. When the weight repetitively moved, the participants perceived a stronger intention compared with a single movement.

For the target positions $u a \_s i d e$ and $o p \_s i d e$, the effect of the trajectory was also significant. Post-hoc comparisons using the Tukey HSD indicated that the perceived intentions for the direct path to the ua_side position was weaker than that for the indirect path in which the weight passed the front position (ua_side-indirect $1 p=.014$ ). For the $o p \_s i d e$ position, the perceived intentions for the direct path were also weaker than that for an indirect path in which the weight passed either the front (op_side-indirect $1 p=.054$ ) or ua_side (op_sideindirect $2 p=.015$ ) position.

\section{Design Recommendations based on Parameter Results}

Below, we discuss the obtained parameter results and summarize design recommendations for weight shift patterns. We also report on an additional analysis about correlations between perceived emotions and intentions. 
To express pleasure, move the weight quickly to the front: With the target position front, significant parameters were found for all three emotional states and intentions. However, with the other two target positions, ua_side and op_side, we did not find any significant parameter for the pleasure perception. The results suggest that the participants' perception of pleasure was significantly affected by the speed parameter, and therefore controlling the speed parameter is presumably effective for designing a weight shift that exhibits a pleasure emotion. However, the results also suggest that slow and repetitive weight shift patterns may cause displeasure depending on the trajectory, and we have to be careful for these patterns.

To express arousal, apply centrifugal force repetitively: Designing a trajectory is important. To express that the robot is aroused, the weight should pass through the ua_side or $o p \_$side positions to make the robot sway in the left-right direction. Furthermore, exhibiting centrifugal force by controlling the rotational movement of the weight, together with controlling the repetition parameter, is presumably effective for producing perceptions of high arousal states. Particularly, in case of a large centrifugal force, if the weight moves to the ua_side or $o p \_s i d e$ position rapidly, the perception of arousal can be enhanced.

To express dominance, use an indirect/long trajectory: The results suggest that taking indirect trajectories is presumably effective for producing perceptions of high dominance states. Specifically, the use of long paths to the target position can enhance the dominance; the results showed that the perceived dominance for the op_side-indirect 2 and the $u a \_s i d e-$ indirect 2 patterns (the longest paths tested) was greater than that for the other conditions. By contrast, if we want to design a weight shift that exhibits a low dominance or submissive emotion, having a direct trajectory with a slow speed would be a nice approach. In addition, fast weight shifts can enhance the perception of dominance when the weight moves to the target positions ua_side or op_side, and repetitive motions can also be effective for the $o p \_$side position.

Perception of intentions maybe enhanced by repetition and indirect trajectories: To indicate that a robotic gadget is attempting to express something (designing intentionality), controlling the repetition is presumably effective. If the gadget repeats a series of continuous movements, then this may help conveying intentions to the users. This finding is consistent with previous reports on intentional movements [41]. Moreover, because the effects of the direct path to the target positions $u a \_s i d e$ and $o p \_s i d e$ were weaker than those of indirect paths, choosing an indirect path maybe better to enhance the gadget's capability of conveying intentions.

The front target position maybe the first choice in conveying intentions that are associated with various emotional states: For each target position, a multiple regression was calculated to predict the perceived intentions based on pleasure, arousal, and dominance. Significant regression equations were found for each target position (front $R^{2}=.19, F(3,201)=$ $15.6, p<.001 ;$ ua_side $R^{2}=.12, F(3,192)=8.75, p<.001$; $o p \_$side $\left.R^{2}=.17, F(3,199)=13.9, p<.001\right)$. For the target position front, all of the dimensions of the emotional states pre- dicted the perceived intentions (pleasure $\beta=-.16, p=.014$; arousal $\beta=.23, p=.002$; dominance $\beta=.21, p=.005$ ). However, only the arousal dimension predicted the perceived intentions for target positions $u a \_$side $(\beta=.26, p<.001)$ and $o p \_s i d e(\beta=.45, p<.001)$. These results suggest that designing the weight shifts such that the weight moves to the front position maybe effective to ensure that the user perceives the various emotions and recognizes the intentions of the robotic gadget in trying to convey those emotions. Similarly, the arousal emotion could be well perceived with intentionality in case where the target position is ua_side or op_side.

\section{Identifying the Semantics of Weight Shift Patterns}

We analyzed how each of the weight shift patterns can represent emotions, with a particular focus on denoting the haptic stimuli, by using the emotion-denoting terms proposed by [37]. In [37], 151 words were defined as the 3D coordinates of pleasure, arousal, and dominance (PAD), and we referred to the positional relationships between those words and the ratings of the emotional states for each condition to determine the semantics of each weight shift pattern. However, the dominance dimension in the PAD model is often omitted (e.g., [47, 49]); thus, we applied the P-A dimensions for this analysis.

For this purpose, we first filtered only conditions with a small dispersion in ratings for pleasure and arousal. For the index, the average distance from a centroid $(\bar{d})$ was used. We also performed a cluster analysis using the k-means method to explore conditions in which two different interpretations are observed among the participants. The pseudo $F$ index [5] was used to describe the ratio of between-cluster variance to within-cluster variance. ${ }^{5}$ A large pseudo $F$ value indicates that the dispersion within a cluster is small and that the distance between clusters is large.

Furthermore, we attempted to denote the intentions of OMOY by referring to the scores of perceived intentions and the participants' descriptions for each weight shift pattern, which were obtained through the elicitation interview procedure.

\section{Single-cluster analysis}

We consider the four conditions (i.e., approximately top $10 \%$ ) with the smallest $\bar{d}$, which were front-indirect2-fast-single $(\bar{d}=1.18)$, ua_side-direct-fast-repetitive $(\bar{d}=1.27)$, frontindirect 1 -slow-repetitive $(\bar{d}=1.38)$, and op_side-direct-fastsingle $(\bar{d}=1.49){ }^{6}$

The centroid and perceived intentions of front-indirect2-fastsingle, ua_side-direct-fast-repetitive, and op_side-direct-fastsingle had the smallest, second-smallest, and fourth-smallest $\bar{d}$ values at (pleasure, arousal, intentions $)=(5.00,5.00,3.75)$, $(4.94,5.76,3.88),(4.65,4.35,3.17)$. These values indicate that neutral emotions and weak intentions were perceived for

${ }^{5}$ The equation was given as follows:

$$
\text { Pseudo } F=\frac{B G S S}{K-1} / \frac{W G S S}{N-K} .
$$

(BGSS: between-group sum of squares, WGSS: within-group sum of squares, $N$ : number of samples, $K$ : number of clusters)

${ }^{6}$ The values of $\bar{d}$ for all conditions are provided in Auxiliary Material for this paper. 


\section{front-indirect1-slow-repetitive}
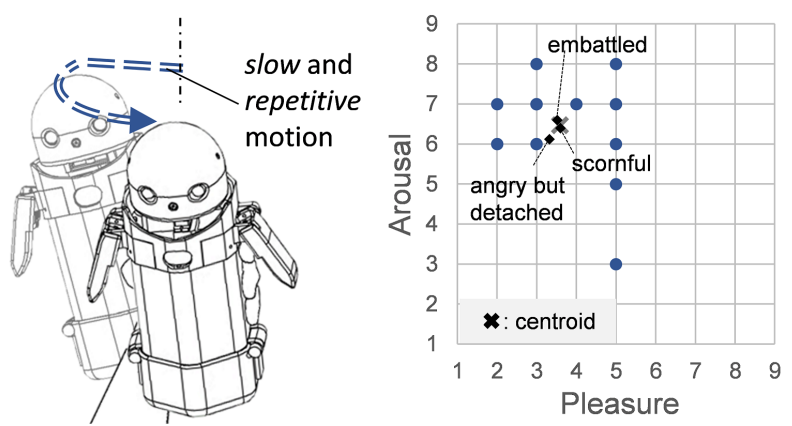

Figure 6. (Left) Behavior of OMOY for the weight shift pattern frontindirect1-slow-repetitive, which had the third-smallest $\bar{d}$ value. (Right) Raw data, the centroid, and the emotion-denoting terms that are the closest to the centroid.

the weight shift patterns. In addition, in these weight shift patterns, participants did not mention anything related to the emotion-denoting terms [37] which were matched around the centroids. Thus, we labeled these weight shift patterns as "unintentional movements with neutral emotion."

For the weight shift pattern front-indirect1-slow-repetitive, the centroid and perceived intentions were scored as (pleasure, arousal, intentions $)=(3.59,6.59,5.76)$, and the emotiondenoting term [37] located closest to the centroid was "embattled" (Figure 6). One participant commented that "(The robot) looks a little uncomfortable", and $65 \%$ of the participants commented that the robot was trying to express such a feeling to the user: for example, "I felt like the robot was complaining to me." and "I felt strongly denied by the robot, and it was also pressing for my answers." Therefore, this weight shift pattern can indicate an embattled state, and may also be effective for intentionally conveying the emotion to the user.

\section{Two-cluster analysis}

The k-means clustering and index results indicated that the occurrence of opposing opinions on the perception of emotions. The four conditions with the largest pseudo $F$ values were $u a \_s i d e$-indirect1-fast-repetitive ( $p$ seudo $F=60.4$ ), $u a$ side-indirect1-slow-repetitive ( $p$ seudo $F=27.4$ ), ua_sideindirect2-fast-repetitive (pseudo $F=26.8$ ), and op_sideindirect2-fast-repetitive ( $p$ seudo $F=25.7$ ). ${ }^{7}$

Ua_side-indirect1-fast-repetitive had the largest pseudo $F$ value, and we gained two clusters (top of Figure 7). Near the center of the first cluster (blue dots in top of Figure 7), "fearful", "terrified", and "hate" terms [37] were plotted, and $78 \%$ of the responses for this cluster interpreted the weight shift as an intentional behavior for expressing a strong refusal: for example, "It seems like a movement in which children are refusing something by throwing a tantrum." or "He is trying to escape from the hand by bending his back." In addition, some participants perceived the intentions of conveying an urgent message to the user: "(The robot is) trying to convey an urgent message to me." or "It seems to convey an alarm. He

\footnotetext{
${ }^{7}$ The values of pseudo $F$ for all conditions are provided in Auxiliary Material for this paper.
}

\section{ua_side-indirect1-fast-repetitive}
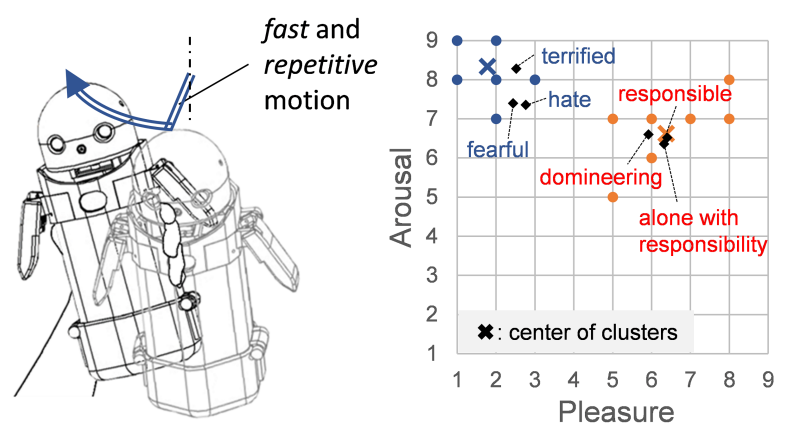

ua_side-indirect1-slow-repetitive

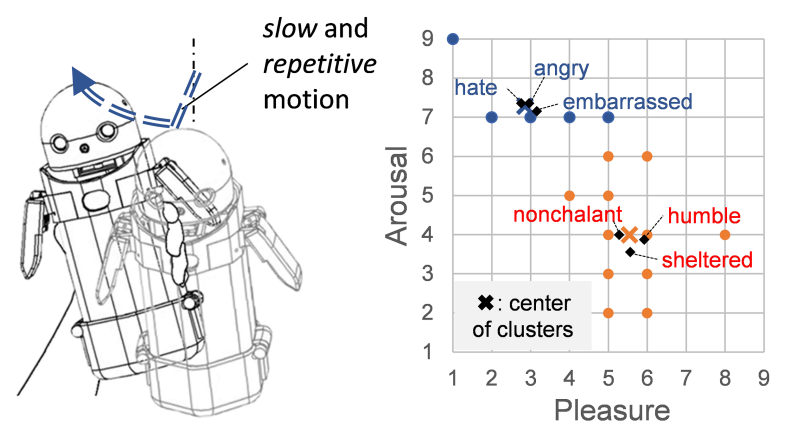

Figure 7. (Top left) Behavior of OMOY for the weight shift pattern ua_side-indirect1-fast-repetitive, which had the largest pseudo $F$ value. (Bottom left) Behavior of OMOY for the weight shift pattern ua_sideindirect1-slow-repetitive, which had the second largest pseudo $F$ value. (Top and bottom right) Raw data for each condition, cluster centers, and the emotion-denoting terms closest to the center of each cluster.

is indicating something urgent, like an earthquakes." Therefore, the first cluster can be denoted as a weight shift pattern for conveying messages with strong refusal or urgency, with strong intentions. By contrast, in the second cluster (red dots in top of Figure 7), no comments are related to the emotiondenoting terms. However, $50 \%$ of the participants perceived the intentions of OMOY as trying to express interest or joy: for example, "It feels like a dog running towards what he is interested by taking the lead." or "He is expressing happiness and gratitude." Based on the center of the cluster and the participants' descriptions, this cluster can be denoted as an emotional state with intermediate pleasure and arousal levels, which are sometimes represented as interest or joy.

Ua_side-indirect1-slow-repetitive had the second largest pseudo $F$ value; this weight shift pattern was a slower version of the pattern with the largest pseudo $F$ value. Near the center of the first cluster, "angry", "hate", and "embarrassed" terms [37] were plotted (bottom in Figure 7), and some of the participants' descriptions were related to these emotions: for example, "The robot was trying to run away from being tickled." or "I think the robot was trembling slightly, and it was frightened." In this cluster, $71 \%$ of the participants perceived intentions, but each participant had a different interpretation of the stimulus. Thus, this cluster can be denoted as an expression for displeasure and arousal states, such as "angry", 
"hate", and "embarrassed", with the feeling that OMOY is trying to express something to the participants. In the second cluster, $55 \%$ of the participants did not perceive the intentions of OMOY, but many of them compared this weight shift to the behavior of small animals: "It feels like a pet getting close to my hand." or "If I compare it to a cat, it feels like it is purring." By contrast, the participants who perceived intentions commented that the intentions were to attract attention to OMOY: for example, "It seems that he wants my attention, maybe he wants to talk with me. But, he is calm and seems like an adult." Considering the center of the cluster, the emotion-denoting terms, and the participants' comments, this cluster can be likened to the behavior of small animals that express pleasure and calm states and may sometimes be useful for attracting the attention of the user.

The weight shift patterns ua_side-indirect2-fast-repetitive and op_side-indirect2-fast-repetitive, which had the third and fourth largest pseudo $F$ values, showed similar cluster plots (Figure 8). These patterns were symmetrical with respect to the target position and trajectory, and the speed and repetition were the same. The centers of the first clusters in these conditions are close to each other, and the participants' comments were also similar: for example, "The robot is feeling uncomfortable, but I don't know what it wants to say. It was trying to tell me something." or "The robot is quite stressed and complaining (me or someone)." In addition, several participants described these movements as a struggle with stress, such as " a movement of a rat running rampage in a bag." Therefore, based on the center of the cluster, the emotion-denoting terms, and the participants' comments, these clusters can be denoted as an explosive action that strongly conveys emotions of "terrified", "fearful", "hate", and "angry" [37] to the user.

For $u a$ side-indirect2-fast-repetitive, the emotion-denoting term [37] closest to the center of second cluster was "aroused", and we obtained participant comments related to that term: for example, "It feels like a dog is coming to me with joy and excitement.” By contrast, for op_side-indirect2-fast-repetitive, no emotion-denoting terms related to the comments are plotted in Figure 8. However, for this weight shift pattern, $80 \%$ of the participants perceived the intentions of OMOY as trying to express joy or happy emotions to the participants: for example, "It seems to be running and playing around my foot to convey its pleasant feeling." or "He is excited, and happy or he wants to jump or he wants to play." In this condition, the percentage of the participants who perceived intentions was higher than the case with $u a \_s i d e$-indirect2-fast-repetitive (44\%). Therefore, the second clusters of both conditions can be denoted as weight shift patterns that express joy or excitement, but the latter may also convey a powerful intention that the gadget is trying to express such emotions to the user.

Overall, we identified weight shift patterns that can express specific emotions and intentions. For some weight shift patterns, opposing interpretations were found among the participants. The meanings represented by these weight shifts are expected to be clear and uniquely defined when combined with other modalities, such as hand gestures, facial expressions, and speech dialogues.

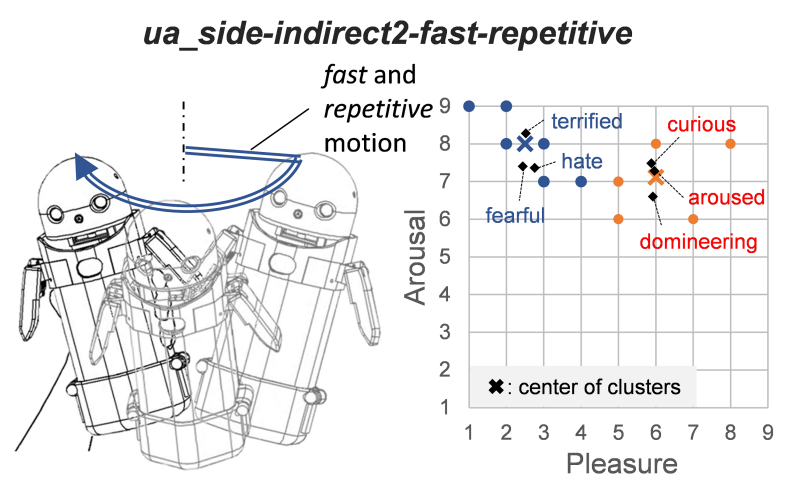

op_side-indirect2-fast-repetitive

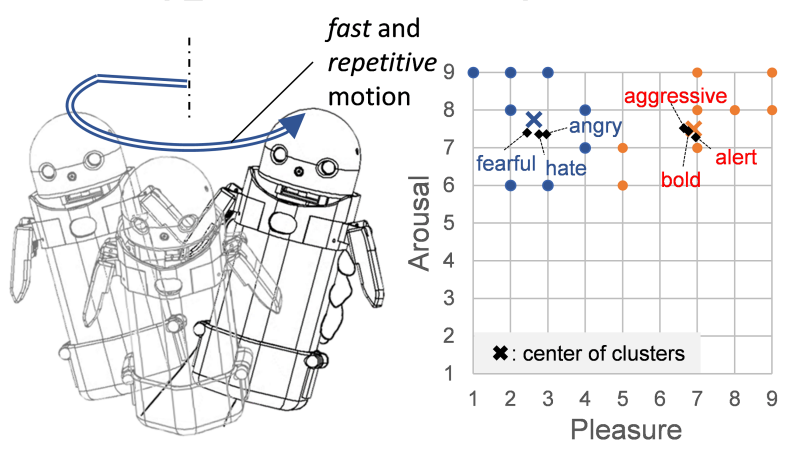

Figure 8. (Top left) Behavior of OMOY for the weight shift pattern ua_side-indirect2-fast-repetitive, which had the third largest pseudo $F$ value. (Bottom left) Behavior of OMOY for the weight shift pattern op_side-indirect2-fast-repetitive which had the forth largest pseudo $F$ value. (Top and bottom right) Raw data for each condition, cluster centers, and the emotion-denoting terms closest to the center of each cluster.

\section{LIMITATIONS, FUTURE WORKS, AND APPLICATIONS}

\section{Contextual Factors}

In this study, we did not provide participants with a detailed narrative frame for OMOY (we just introduced it to the participants as a communication robot). As was discussed in previous literature [4], human perception on robot emotions is sensitive to narrative framing factors. In this study, we attempted to collect the open perceptions of the participants by not specifying the narrative frame; however, the results have to be interpreted with considering the potential effect caused by the narrative framing factors.

According to the affective grounding perspective that was introduced in human-robot interaction literature [19], "emotion (how behavior is interpreted emotionally) and emotion regulation (how the emotional meaning of behavior is re-adjusted through repair) are not only internal processes but that can be seen as interpersonal processes that are jointly coordinated by participants of an interaction." As mentioned in the previous paragraph, we did not clearly specify the context behind the robot. Therefore, participants could have experienced a difficulty in interpreting the robot's emotions, which may be viewed as an initial phase in the affective grounding. Among a total of 648 cases, there were 29 cases in which some of the participants reported such difficulties and could not answer to the questionnaire items. In 12 of those cases, the partic- 
ipants commented that they perceived purely mechanical or inanimate movements without any sort of biological agencies, such as "it feels like a rugby ball bouncing," "it was like a machine changing its gear," or "HDD (hard disk drive) noise." In other 10 cases, the participants felt that the movements were too complex to make any comment on. Finally, the other seven cases include four cases reporting malfunctions (in fact not broken) and three cases commenting that the movements were too small to grasp them. It will be an interesting future research to investigate how those interpretations are changing or re-adjusted through the continuous use of OMOY across multiple situations.

\section{Qualitative Analysis}

Related to the content that was discussed in the previous paragraphs, an important future work is to investigate the participants' open comments by using qualitative analysis methods. So far, we have confirmed that their descriptions can be classified into, at least, (1) emblems, (2) illustrators [11], (3) cognitive processes, and (4) impressions for subtle posture changes that were caused by weight shifts. It would likely be necessary to make use of multiple qualitative analysis methods to deal with all those descriptions.

\section{Other Limitations}

We have to report several considerations with respect to the scalability of our study results. The results of this study were based on the characteristics of our prototype; thus, the results may not hold under changes in parameters of the gadget, such as the appearance, shape, and materials. For future works, we are interested in testing the weight shift functions of different gadgets, for example, by testing a gadget with a simpler appearance, such as a cylinder or cubes, or another gadget with a more human-like appearance, such as a baby doll.

In addition, the number of attributes for each parameter in the elicitation study may not be sufficient. For example, we did not test on ease in/out movements. In our setting, it seemed difficult to distinguish small velocity variations; however, with bigger gadgets, they could be perceived. Because the goal of our study was to explore the effective parameters for weight shifts, we set two or three attributes for each parameter. To strengthen our results, a follow-up study is needed to investigate the effects of each parameter more in detail.

Human factors, such as gender differences or personality traits, should be considered. In particular, the evaluations for social robots can differ depending on the personality traits of the user [48].

The servo noise of the robot may have affected the results. In this study, the movements of the actuator were completely invisible. To compensate for the lack of visual cues in assessing the emotional state and intentions of OMOY with other cues, the participants may have become sensitive to the noise factor (audio cues). In the elicitation study, a few participants mentioned an evaluation bias due to the servo noise. Verifying the effect of servo noise maybe necessary or can be an interesting topic in future works.

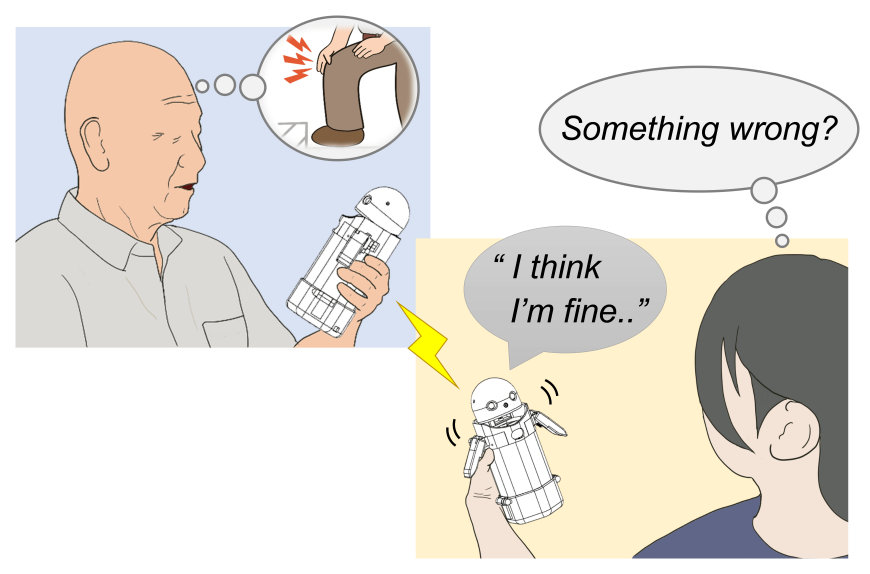

Figure 9. A future use case. OMOY could be used as a social mediator for humans. It could help people sending/receiving messages that contain subtle but important information that is sometimes not so easy to express by words or voices. For example, we assume remote communication involving elderly people who live separately from his/her family members.

\section{Vision for the Future: Social Mediator Robot}

Considering the future application of this study, the first thing we have to do next is to study the effects of weight shifts combined with other robotic behaviors such as gestures or speech dialogues. We aim for a scenario in which a robotic gadget serves as a social mediator that facilitates human communication (Figure 9). One use case is remote messaging between elderly people and their family members who live separately. To prevent elderly people from being socially isolated, the role of messaging is becoming very important. However, sometimes it is difficult for elderly people to reveal their true emotions [8, $23]$. On the other hand, a recent study reported that a social robot could well facilitate the self-disclosure of elderly people in remote communication [30, 31]. Therefore, we expect that a robotic gadget equipped with the function of enhancing messages by using weight shifts, gestures, and speech dialogues would provide a promising solution for these people.

\section{CONCLUSION}

In this paper, we presented the design of a robotic gadget that exhibits weight shifts by controlling a movable weight inside its body. Results from an elicitation study were reported with detailed analyses about four movement parameters and users' perceptions. The semantics of weight shift patterns were also investigated. These findings and summarized knowledge provided design recommendations for weight shifts that help the development of robotic gadgets that enhance messaging emotionally and intentionally between people.

\section{ACKNOWLEDGMENTS}

This work was supported by JSPS KAKENHI Grant Number 19H01112. We appreciate the participants of this study for their cooperation and valuable feedback. We also appreciate the program committee members and reviewers for their efforts in reviewing this paper and providing us with important comments. 


\section{REFERENCES}

[1] Ahmad Hoirul Basori, Abdullah Bade, Mohd Shahrizal Sunar, Daut Daman, and Nadzari Saari. 2009. Haptic Vibration for Emotional Expression of Avatar to Enhance the Realism of Virtual Reality. In Proceedings of the International Conference on Computer Technology and Development. IEEE, 416-420. DOI : http://dx.doi.org/10.1109/ICCTD. 2009.132

[2] Alexis E. Block and Katherine J. Kuchenbecker. 2018. Emotionally Supporting Humans Through Robot Hugs. In Companion of the 2018 ACM/IEEE International Conference on Human-Robot Interaction. ACM, 293-294. DOI :

http://dx.doi.org/10.1145/3173386.3176905

[3] Margaret M. Bradley and Peter J. Lang. 1994. Measuring Emotion: The Self-Assessment Manikin and the Semantic Differential. Journal of Behavior Therapy and Experimental Psychiatry 25, 1 (1994), 49-59. DOI : http://dx.doi .org/10.1016/0005-7916(94)90063-9

[4] Paul Bucci, Lotus Zhang, Xi Laura Cang, and Karon E. MacLean. 2018. Is It Happy?: Behavioural and Narrative Frame Complexity Impact Perceptions of a Simple Furry Robot's Emotions. In Proceedings of the 2018 CHI Conference on Human Factors in Computing Systems. ACM, Paper No.509. DOI :

http://dx.doi.org/10.1145/3173574. 3174083

[5] Tadeusz Caliński and Jerzy Harabasz. 1974. A Dendrite Method for Cluster Analysis. Communications in Statistics 3, 1 (1974), 1-27. DOI :

http://dx.doi.org/10.1080/03610927408827101

[6] Angela Chang, Sile O’Modhrain, Rob Jacob, Eric Gunther, and Hiroshi Ishii. 2002. ComTouch: Design of a Vibrotactile Communication Device. In Proceedings of the 4th Conference on Designing Interactive Systems: Processes, Practices, Methods, and Techniques. ACM, 312-320. DOI :

http://dx.doi.org/10.1145/778712.778755

[7] Sharp Corporation. 2016. RoBoHoN. (2016). Retrieved September 2, 2019 from https://robohon. com/.

[8] Nikolas Coupland, Justine Coupland, Howard Giles, and Karen Henwood. 1988. Accommodating the Elderly: Invoking and Extending a Theory. Language in Society 17, 1 (1988), 1-41. DOI :

http://dx.doi.org/10.1017/S0047404500012574

[9] Maartje M.A. de Graaf, Somaya Ben Allouch, and Jan A.G.M. van Dijk. 2016. Long-Term Acceptance of Social Robots in Domestic Environments: Insights from a User's Perspective. In Proceedings of the 2016 AAAI Spring Symposium Series: Enabling Computing Research in Socially Intelligent Human-Robot Interaction. AAAI, 96-103.

[10] Winand H Dittrich and Stephen E G Lea. 1994. Visual Perception of Intentional Motion. Perception 23, 3 (1994), 253-268. DOI :

http://dx.doi.org/10.1068/p230253
[11] Paul Ekman and Wallace V. Friesen. 1969. The Repertoire of Nonverbal Behavior: Categories, Origins, Usage, and Coding. Semiotica 1, 1 (1969), 49-98. DOI : http://dx.doi.org/10.1515/semi.1969.1.1.49

[12] Frank A. Geldard. 1960. Some Neglected Possibilities of Communication. Science 131, 3413 (1960), 1583-1588. DOI : http://dx. doi .org/10.1126/science.131.3413.1583

[13] Rachel Gockley, Allison Bruce, Jodi Forlizzi, Marek Michalowski, Anne Mundell, Stephanie Rosenthal, Brennan Sellner, Reid Simmons, Kevin Snipes, Alan C. Schultz, and Jue Wang. 2005. Designing Robots for Long-term Social Interaction. In Proceedings of the IEEE/RSJ International Conference on Intelligent Robots and Systems. IEEE, 2199-2204. DOI : http://dx.doi .org/10.1109/IROS.2005.1545303

[14] Fumio Hara and Hiroshi Kobayashi. 1996. A Face Robot able to Recognize and Produce Facial Expression. In Proceedings of the IEEE/RSJ International Conference on Intelligent Robots and Systems. IEEE, 1600-1607. DOI : http://dx.doi .org/10.1109/IROS. 1996.569026

[15] Fabian Hemmert, Susann Hamann, Matthias Löwe, Josefine Zeipelt, and Gesche Joost. 2010. Weight-Shifting Mobiles: Two-Dimensional Gravitational Displays in Mobile Phones. In CHI 2010 Extended Abstracts on Human Factors in Computing Systems. ACM, 3087-3092. DOI : http://dx.doi.org/10.1145/1753846.1753922

[16] Yuhan $\mathrm{Hu}$, Zhengnan Zhao, Abheek Vimal, and Guy Hoffman. 2018. Soft Skin Texture Modulation for Social Robotics. In Proceedings of the 2018 IEEE International Conference on Soft Robotics. IEEE, 182-187. DOI : http://dx.doi.org/10.1109/ROBOSOFT . 2018.8404917

[17] Eun-Sook Jee, Yong-Jeon Cheong, Chong Hui Kim, Dong-Soo Kwon, and Hisato Kobayashi. 2009. Sound Production for the Emotional Expression of Socially Interactive Robots. In V. Kulyukin (eds) Advances in Human-Robot Interaction. IntechOpen, 257-272. DOI : http://dx.doi.org/10.5772/6838

[18] Nils B. Jostmann, Daniël Lakens, and Thomas W. Schubert. 2009. Weight as an Embodiment of Importance. Psychological Science 20, 9 (2009), 1169-1174. DOI : http://dx.doi.org/10.1111/j.1467-9280.2009.02426.x

[19] Malte F. Jung. 2017. Affective Grounding in Human-Robot Interaction. In Proceedings of the 2017 ACM/IEEE International Conference on Human-Robot Interaction. ACM, 263-273. DOI : http://dx.doi.org/10.1145/2909824.3020224

[20] Lawrence H. Kim and Sean Follmer. 2019. SwarmHaptics: Haptic Display with Swarm Robots. In Proceedings of the 2019 CHI Conference on Human Factors in Computing Systems. ACM, Paper No.688. DOI : http://dx.doi .org/10.1145/3290605.3300918 
[21] Diana Löffler, Nina Schmidt, and Robert Tscharn. 2018. Multimodal Expression of Artificial Emotion in Social Robots Using Color, Motion and Sound. In Proceedings of the ACM/IEEE International Conference on Human-Robot Interaction. ACM, 334-343. DOI : http://dx.doi.org/10.1145/3171221.3171261

[22] Michal Luria, Jessica Hodgins, and Jodi Forlizzi. 2018. The Effects of Eye Design on the Perception of Social Robots. In Proceedings of the 27th IEEE International Conference on Robot and Human Interactive Communication. IEEE, 1032-1037. DOI : http://dx.doi.org/10.1109/ROMAN.2018.8525767

[23] Stephen M. Marson and Rasby M. Powell. 2014. Goffman and the Infantilization of Elderly Persons: a Theory in Development. Journal of Sociology and Social Welfare 41, 4 (2014), 143-158.

[24] Albert Mehrabian. 1996. Pleasure-Arousal-Dominance: A General Framework for Describing and Measuring Individual Differences in Temperament. Current Psychology 14, 4 (1996), 261-292. DOI:

http://dx.doi .org/10.1007/BF02686918

[25] Toru Nakata, Tomomasa Sato, and Taketoshi Mori. 1998. Expression of Emotion and Intention by Robot Body Movement. In Y. Kakazu, M. Wada, T. Sato (eds) Intelligent Autonomous System IAS-5. IOS Press, 352-359.

[26] Jeffrey S. Nevid. 2018. Essentials of Psychology: Concepts and Applications, 5th Edition. Cengage.

[27] Jiaqi Nie, Michelle Park, Angie Lorena Marin, and S. Shyam Sundar. 2012. Can You Hold My Hand?: Physical Warmth in Human-Robot Interaction. In Proceedings of the 7th ACM/IEEE International Conference on Human-Robot Interaction. ACM, 201-202. DOI :

http://dx.doi.org/10.1145/2157689.2157755

[28] Ryuma Niiyama, Lining Yao, and Hiroshi Ishii. 2014. Weight and Volume Changing Device with Liquid Metal Transfer. In Proceedings of the 8th International Conference on Tangible, Embedded and Embodied Interaction. ACM, 49-52. DOI : http://dx.doi .org/10.1145/2540930.2540953

[29] Hiroshi Noguchi, Yuiko Koyano, Hiromi Mori, Chieko Komiyama, Hiromi Sanada, and Taketoshi Mori. 2019. Exploration of Communication Robot Use for Older Patients in an Acute Hospital based on Case Trials. Journal of Nursing Science and Engineering 6, 2 (2019), 70-82. DOI : http://dx.doi.org/10.24462/jnse.6.2_70

[30] Yohei Noguchi, Hiroko Kamide, and Fumihide Tanaka. 2018. Effects on the Self-disclosure of Elderly People by Using a Robot Which Intermediates Remote Communication. In Proceedings of the 27th IEEE International Conference on Robot and Human Interactive Communication. IEEE, 612-617. DOI : http://dx.doi.org/10.1109/ROMAN. 2018.8525562

[31] Yohei Noguchi, Hiroko Kamide, and Fumihide Tanaka. 2020. Personality Traits for Social Mediator Robot
Encouraging Elderly Self-Disclosure on Loss Experiences. ACM Transactions on Human-Robot Interaction (2020), in press.

[32] Marianna Obrist, Sue Ann Seah, and Sriram Subramanian. 2013. Talking about Tactile Experiences. In Proceedings of the SIGCHI Conference on Human Factors in Computing Systems. ACM, 1659-1668. DOI : http://dx.doi .org/10.1145/2470654.2466220

[33] Denis Peña and Fumihide Tanaka. 2018. Validation of the Design of a Robot to Study the Thermo-Emotional Expression. In Ge S. et al. (eds) Social Robotics. ICSR 2018. Lecture Notes in Computer Science, vol.11357. Springer, Cham, 75-85. DOI :

http://dx.doi.org/10.1007/978-3-030-05204-1_8

[34] Shafiq Ur Réhman. 2010. Expressing Emotions through Vibration for Perception and Control. Ph.D. Dissertation. Department of Applied Physics and Electronics, Umeå University.

[35] Shafiq Ur Réhman and Li Liu. 2008. Vibrotactile Emotions on a Mobile Phone. In Proceedings of the IEEE International Conference on Signal Image Technology and Internet Based Systems. IEEE, 239-243. DOI : http://dx.doi.org/10.1109/SITIS.2008.72

[36] Shafiq Ur Réhman and Li Liu. 2010. iFeeling: Vibrotactile Rendering of Human Emotions on Mobile Phones. In Jiang X., Ma M.Y., Chen C.W. (eds) Mobile Multimedia Processing. WMMP 2008. Lecture Notes in Computer Science, vol.5960. Springer, Berlin, Heidelberg, 1-20. DOI:

http://dx.doi .org/10.1007/978-3-642-12349-8_1

[37] James A. Russell and Albert Mehrabian. 1977. Evidence for a Three-factor Theory of Emotions. Journal of Research in Personality 11, 3 (1977), 273-294. DOI: http://dx . doi .org/10. 1016/0092-6566(77)90037-X

[38] Jotaro Shigeyama, Takeru Hashimoto, Shigeo Yoshida, Taiju Aoki, Takuji Narumi, Tomohiro Tanikawa, and Michitaka Hirose. 2018. Transcalibur: Weight Moving VR Controller for Dynamic Rendering of 2D Shape Using Haptic Shape Illusion. In Proceedings of the ACM SIGGRAPH Emerging Technologies. ACM, Article No.19. DOI : http://dx.doi.org/10.1145/3214907.3214923

[39] Jotaro Shigeyama, Takeru Hashimoto, Shigeo Yoshida, Takuji Narumi, Tomohiro Tanikawa, and Michitaka Hirose. 2019. Transcalibur: A Weight Shifting Virtual Reality Controller for 2D Shape Rendering Based on Computational Perception Model. In Proceedings of the 2019 CHI Conference on Human Factors in Computing Systems. ACM, Paper No.11. DOI: http://dx.doi.org/10.1145/3290605.3300241

[40] Sichao Song and Seiji Yamada. 2017. Expressing Emotions through Color, Sound, and Vibration with an Appearance-Constrained Social Robot. In Proceedings of the ACM/IEEE International Conference on Human-Robot Interaction. ACM, 2-11. DOI: http://dx.doi .org/10.1145/2909824.3020239 
[41] Barbette Spitler. 2016. Make It Intentional: Harness the Power of Positive Perspectives. Balboa Press.

[42] Colin Swindells, Alex Unden, and Tao Sang. 2003. TorqueBAR: An Ungrounded Haptic Feedback Device. In Proceedings of the 5th International Conference on Multimodal Interfaces. ACM, 52-59. DOI: http://dx.doi.org/10.1145/958432.958445

[43] Hong Z. Tan, Nathaniel I. Durlach, Charlotte M. Reed, and William M. Rabinowitz. 1999. Information Transmission with a Multifinger Tactual Display. Perception \& Psychophysics 61, 6 (1999), 993-1008. DOI : http://dx.doi.org/10.3758/BF03207608

[44] Fumihide Tanaka, Javier R. Movellan, Bret Fortenberry, and Kazuki Aisaka. 2006. Daily HRI Evaluation at a Classroom Environment: Reports from Dance Interaction Experiments. In Proceedings of the 1st ACM SIGCHI/SIGART Conference on Human-Robot Interaction. ACM, 3-9. DOI : http://dx.doi.org/10.1145/1121241.1121245

[45] Kazunori Terada, Atsushi Yamauchi, and Akira Ito. 2012. Artificial Emotion Expression for a Robot by Dynamic Color Change. In Proceedings of the 21st IEEE International Symposium on Robot and Human Interactive Communication. IEEE, 314-321. DOI : http://dx.doi.org/10.1109/ROMAN . 2012.6343772

[46] Takayuki Todo. 2018. SEER: Simulative Emotional Expression Robot. In Proceedings of the ACM
SIGGRAPH Emerging Technologies. ACM, Article No.15. DOI : http://dx.doi.org/10.1145/3214907.3214921

[47] Graham Wilson, Dobromir Dobrev, and Stephen A. Brewster. 2016. Hot Under the Collar: Mapping Thermal Feedback to Dimensional Models of Emotion. In Proceedings of the 2016 CHI Conference on Human Factors in Computing Systems. ACM, 4838-4849. DOI : http://dx.doi.org/10.1145/2858036.2858205

[48] Sarah Woods, Kerstin Dautenhahn, Christina Kaouri, René te Boekhorst, and Kheng Lee Koay. 2005. Is This Robot Like Me? Links between Human and Robot Personality Traits. In Proceedings of the 5th IEEE-RAS International Conference on Humanoid Robots. IEEE, 375-380. DOI :

http://dx.doi.org/10.1109/ICHR. 2005.1573596

[49] Steve Yohanan and Karon E. MacLean. 2011. Design and Assessment of the Haptic Creature's Affect Display. In Proceedings of the 6th International Conference on Human-Robot Interaction. ACM, 473-480. DOI : http://dx.doi.org/10.1145/1957656.1957820

[50] André Zenner and Antonio Krüger. 2017. Shifty: A Weight-Shifting Dynamic Passive Haptic Proxy to Enhance Object Perception in Virtual Reality. IEEE Transactions on Visualization and Computer Graphics 23, 4 (2017), 1285-1294. DOI :

http://dx.doi.org/10.1109/TVCG. 2017.2656978 